\title{
Dysregulated LIGHT expression on T cells mediates intestinal inflammation and contributes to IgA nephropathy
}

\author{
Jing Wang, ${ }^{1}$ Robert A. Anders, ${ }^{1}$ Qiang Wu, ${ }^{2}$ Dacheng Peng, ${ }^{1}$ Judy H. Cho, ${ }^{3}$ Yonglian Sun, ${ }^{1}$ \\ Reda Karaliukas, ${ }^{3}$ Hyung-Sik Kang, ${ }^{1}$ Jerrold R. Turner, ${ }^{1}$ and Yang-Xin Fu ${ }^{1}$ \\ ${ }^{1}$ Department of Pathology, University of Chicago, Chicago, Illinois, USA. ${ }^{2}$ Department of Dermatology, University of lowa, Iowa City, Iowa, USA. \\ ${ }^{3}$ Department of Medicine, University of Chicago, Chicago, Illinois, USA.
}

\begin{abstract}
Whether and how $\mathrm{T}$ cells contribute to the pathogenesis of immunoglobulin A nephropathy (IgAN) has not been well defined. Here, we explore a murine model that spontaneously develops $T$ cell-mediated intestinal inflammation accompanied by pathological features similar to those of human IgAN. Intestinal inflammation mediated by LIGHT, a ligand for lymphotoxin $\beta$ receptor (LT $\beta R$ ), not only stimulates IgA overproduction in the gut but also results in defective IgA transportation into the gut lumen, causing a dramatic increase in serum polymeric IgA. Engagement of LT $\beta$ R by LIGHT is essential for both intestinal inflammation and hyperserum IgA syndrome in our LIGHT transgenic model. Impressively, the majority of patients with inflammatory bowel disease showed increased IgA-producing cells in the gut, elevated serum IgA levels, and severe hematuria, a hallmark of IgAN. These observations indicate the critical contributions of dysregulated LIGHT expression and intestinal inflammation to the pathogenesis of IgAN.
\end{abstract}

\section{Introduction}

Immunoglobulin A (IgA) is the most abundantly produced Ig in mammals and plays an important role in the defense against microorganisms at mucosal surfaces. Mucosal immune responses in the intestine are highly dependent on costimulation (1) and $\mathrm{T}$ lymphocyte help (2-4). The generation of antigen-specific IgA-committed B cells can occur in both Peyer's patches (PPs) (5) and intestinal lamina propria (LP) $(6,7)$, in which there are numerous plasma cells (PCs) producing polymeric IgA (pIgA). Transport of pIgA into the mucosal secretions occurs via the polymeric Ig receptor on the intestinal epithelial cells (8). IgA nephropathy (IgAN), the most common type of human glomerulonephritis (GN) worldwide, is characterized by elevated serum IgA in 50\% of patients and renal mesangial deposition of IgA and complement (9). The molecular mechanism of human IgAN has not been well defined, and the source of pathogenic IgA is under debate.

Previous studies have suggested a basic dysregulation of IgA production by showing increased serum levels of IgA, IgA-containing immune complexes, and PIgA, and enhanced IgA-specific T helper activity in human patients $(10-12)$. T cells are implicated in the pathogenesis of this disorder; however, the direct role of $\mathrm{T}$ cells in the pathogenesis of IgAN is unclear, as few T cells can be identified in glomerular mesangium. Although the origin of pathogenic pIgA in patients is unclear, the critical role of PIgA in renal deposition and

Nonstandard abbreviations used: alkaline phosphatase (AP); bone marrow (BM); C57BL/6 (B6); fast protein liquid chromatography (FPLC); glomerulonephritis (GN); graft-versus-host disease (GVHD); gut-associated lymphoid tissue (GALT); IgAproducing plasma cell (IgA-PC); immunoglobulin A nephropathy (IgAN); inflammatory bowel disease (IBD); lamina propria (LP); lymphotoxin $\beta$ receptor (LT $\beta R$ ); mesenteric lymph node (MLN); minor histocompatibility (MiHC); monomeric $\operatorname{IgA}(\mathrm{mIgA})$; periodic acid-Schiff (PAS); Peyer's patch (PP); plasma cell (PC); polymeric IgA (pIgA); transgenic $(\mathrm{Tg})$; urine analysis (UA).

Conflict of interest: The authors have declared that no conflict of interest exists. Citation for this article: J. Clin. Invest. 113:826-835 (2004). doi:10.1172/JCI200420096. induction of pathological changes in the glomeruli has been well documented $(13,14)$. Moreover, certain pIgA molecules deposited within the glomerular mesangium are directed against microbial or dietary antigens present in the intestinal mucosa and lumen (15). However, the lack of animal models makes experimental linkage between intestinal inflammation and IgAN inconclusive. The molecular mechanisms by which the impaired mucosal immunity results in the progression of IgA-associated GN are still unknown.

Our previous data demonstrated that the lymphotoxin $\beta$ receptor $(\mathrm{LT} \beta \mathrm{R})$ in the intestine is required for the production of $\operatorname{IgA}$, as $L T \beta R^{-/-}$mice display profoundly low levels of baseline serum and fecal IgA (16). LT $\beta$ R signaling is essential for the proper expression of chemokines and adhesion molecules within the intestinal LP environment and determines the effective homing of IgA-producing PC (IgA-PC) precursors (16). In addition, LT $\beta \mathrm{R}$ is required for the development of mucosal lymphoid organs (17). LIGHT is a ligand for LT $\beta$ R (18) and cooperates with LT $\beta$, another LT $\beta$ R ligand, in mesenteric lymph node (MLN) organogenesis (19). LIGHT is expressed on activated T cells (18) and has a potent costimulatory effect on T cells $(20,21)$. Its upregulation is associated with $\mathrm{T}$ cell-mediated diseases including graft-versus-host disease (GVHD), transplant rejection, and autoimmune diabetes (20-23). We have recently found that constitutive expression of LIGHT on T cells can also induce chemokine production through LT $\beta$ R signaling, leading to the formation of lymphoid tissues in the spleen (24).

We hypothesized that an increase in activated lymphocyte infiltration in the gut, such as that in inflammatory bowel disease (IBD), would cause dysregulated IgA production via LT $\beta$ R signaling, which might serve as a mechanism for the pathogenesis of IgAN. Previous studies have implicated a link between secondary IgAN and IBD $(25-28)$. We found here that IBD patients with active intestinal inflammation showed significantly increased numbers of IgA-producing cells in the gut. These changes were accompanied by elevated serum IgA and hematuria, both hallmarks of IgAN. To dissect if 
and how activated $\mathrm{T}$ cells in the gut contribute to dysregulation of IgA production and to the pathogenesis of IgAN, we analyzed several murine models. Our LIGHT transgenic ( $\mathrm{Tg}$ ) mice develop $\mathrm{T}$ cell-mediated intestinal inflammation, which leads to profound dysregulation of PIgA production and impaired transportation and clearance, leading to dominant mesangial IgA deposition. Importantly, the IgAN phenotype was found to be dependent on LT $\beta$ R signaling via LIGHT. Therefore, our results highlight a direct contribution of T cell-mediated mucosal immunity to IgAN and suggest that LIGHT is a critical player in the pathogenesis of IgAN.

\section{Methods}

Mice. LIGHT Tg mice were generated as previously described (21) and were maintained in specific pathogen-free facility according to University of Chicago policy. $R A G-1^{-/}$(C57BL/6 background), C57BL/6 (B6), and LP/J mice were purchased from The Jackson Laboratory. $L T \beta R^{-/-}$mice were kindly provided by K. Pfeffer (Institute of Medical Microbiology, Immunology, and Hygiene, Technical University of Munich, Munich, Germany). Animal and human experimentation protocols were consistent with NIH guidelines and were approved by the Institutional Animal Care and Use Committee (IACUC) at the University of Chicago.

$A b$ 's and flow cytometry. The following Ab's were used for FACS analysis: FITC-anti-mouse B220 (PharMingen, San Diego, California, USA); biotin-labeled goat anti-mouse IgA (Southern Biotechnology Associates, Birmingham, Alabama, USA); and streptavidinphycoerythrin (Immunotech, France). Cells were analyzed using a FACScan (Becton Dickinson, San Jose, California, USA).

ELISA. Serum levels of IgA, IgG1, and IgG2a, and fecal IgA levels, were measured by a modification of a published protocol for sandwich ELISA (21). Polystyrene microtiter plates (ThermoLabsystems, Franklin, Massachusetts, USA) were coated with unlabeled goat anti-mouse Ig heavy and light chains $(\mathrm{H}+\mathrm{L})(2 \mu \mathrm{g} / \mathrm{ml})$ (Southern Biotechnology Associates). After the plates were blocked with bovine serum albumin (0.1\%), the serum samples were applied at serial dilutions. After incubation of plates, alkaline phosphatase-goat anti-mouse IgA, IgG1, or IgG2a (Southern Biotechnology Associates) was added (1:2,000 dilution) and incubated for 90 minutes. Color was developed by the addition of p-nitrophenyl phosphate solution (Sigma-Aldrich, St. Louis, Missouri, USA), and absorbance was measured at $405 \mathrm{~nm}$. The standard curve was determined by reaction with different concentrations of purified mouse serum IgA, IgG1, and IgG2a (Southern Biotechnology Associates). Fecal samples were collected and dissolved at a concentration of $100 \mathrm{mg} / \mathrm{ml}$ in a buffer of $1 \times \mathrm{PBS}$ and $0.01 \% \mathrm{NaN}_{3}$. For human IgA ELISA, a similar protocol was used except goat anti-human $\operatorname{Ig}(\mathrm{H}+\mathrm{L})(2 \mu \mathrm{g} / \mathrm{ml})$ was the coating antibody and goat anti-human IgA-alkaline phosphatase (AP) was the detecting antibody (1:2,000 dilution) (Southern Biotechnology Associates). Anti-IgA a was purchased from BD (PharMingen, San Diego, California, USA), and IgA a ELISA was performed according to a previous study (29). Detection of anti-DNA antibody was performed as previously described (21). Briefly, 96-well plates were coated with sperm DNA $(250 \mu \mathrm{g} / \mathrm{ml})$ (Sigma-Aldrich). Serial dilutions of serum were applied to the wells and subsequently goat anti-mouse IgA-AP or goat anti-mouse IgG-AP (Southern Biotechnology Associates) were used as detecting antibodies (1:2,000 dilution). OD values were normalized to a serum sample from an MRL/lpr mouse ( 6 months old) used as a standard, and the arbitrary units for serum samples were calculated based on the standard and dilution factor. For detection of human samples, goat anti-human IgG-AP or goat anti-human IgA-AP was used as the detecting antibody (Southern Biotechnology Associates).

Urinalysis. Urine samples were collected from aged WT and Tg mice 6-8 months of age and were subjected to strip analysis (Multistix 10 SG; Bayer Corp., Elkhart, Indiana, USA). Proteinuria was graded as follows: 0 , negative or trace; $1,30 \mathrm{mg} / \mathrm{dl} ; 2,100 \mathrm{mg} / \mathrm{dl} ; 3$, $300 \mathrm{mg} / \mathrm{dl} ; 4,>2,000 \mathrm{mg} / \mathrm{dl}$. Hematuria was graded as follows: 0 , negative; 1 , trace; 2 , moderate. For human patients, standard laboratory protocols were used to detect macroscopic and microscopic hematuria in a clinical reference lab.

Size analysis of serum IgA. Size fractionation of the pooled sera was performed by fast protein liquid chromatography (FPLC) on a 1.0$\mathrm{cm} \times 60-\mathrm{cm}$ column (Tandem of Superose 6 HR 10/30; Amersham Pharmacia Biotech Inc. Piscataway, New Jersey, USA) with a flow rate of $0.4 \mathrm{ml} / \mathrm{min}$. FPLC running buffer contains $0.02 \mathrm{M}$ $\mathrm{NaH}_{2} \mathrm{PO}_{4}, 0.02 \mathrm{M} \mathrm{Na}_{2} \mathrm{HPO} 4$, and $0.05 \mathrm{M} \mathrm{NaCl}$ ( $\mathrm{pH}$ 7.2). Seventy fractions of each sample were collected, and fractions $11-57$ or fractions 16-57 were plotted against IgA concentration. Gel-filtration molecular mass markers were used (Bio-Rad Laboratories, Richmond, California, USA).

Histopathology and immunofluorescence staining. The immunofluorescence staining protocol for $\operatorname{IgA}$ and $\mathrm{C} 3$ in the glomeruli was described previously (21). The following antibodies were used: biotin-labeled goat anti-mouse IgA (Southern Biotechnology Associates), FITC-conjugated rat anti-mouse C3 (Cappel Laboratories, Westchester, Pennsylvania, USA), FITC-conjugated anti-mouse IgG (Caltag Laboratories, Burlingame, California, USA), and FITC-conjugated anti-mouse IgM (BD PharMingen, San Diego, California, USA). MAdCAM-1 staining was done as previously described (24). $\mathrm{H} \& \mathrm{E}$ staining of kidney and intestine samples was performed as described previously (21). The degree and severity of glomerular lesions were assessed by a pathologist "double-blinded" to sample identity. For this, 40 randomly selected glomeruli per kidney from WT or Tg mice ( $n=4$ mice per group) were assigned scores for mesangial deposits as follows: none, focal ( $<50 \%$ of glomeruli), or diffuse ( $>50 \%$ of glomeruli). For immunohistochemical staining of IgA, intestines from control and IBD patients were processed as described previously (21), then were stained with rabbit anti-human IgA (DAKO), developed using the biotin/avidin/DAB Ventana ES system, and counterstained with Harris hematoxylin (SigmaAldrich). The paraffin-embedded murine intestine samples were dewaxed, stained with goat anti-mouse IgA (Southern Biotechnology Associates), horse anti-goat Ig-biotin (Vector Laboratories, Burlingame, California, USA) and ABC-HRP (Vectastain ABC-HRP kit; Vector Laboratories), developed with the DAB system (DAKO), and counterstained with hematoxylin (Sigma-Aldrich).

Bone marrow (BM) and splenocyte transplantation and adoptive transfer of LN cells. Bone marrow (BM) cells and splenocytes were collected from WT B6 mice as described previously, and $2 \times 10^{6} \mathrm{BM}$ cells and $5 \times 10^{7}$ splenocytes were intravenously (i.v.) transferred into LP/J mice that were lethally irradiated (950 rad). LT $\beta \mathrm{R}-\mathrm{Ig}$ was administered in day 0 and day 7. Samples were collected 20 days after BM and splenocyte transfer and were subjected to ELISA and H\&E and immunofluorescence staining. MLN cells were collected from WT littermates or LIGHT Tg mice (2-3 months old) and $6 \times 10^{6} \mathrm{LN}$ cells were i.v. transferred into $R A G-1^{-/-}$mice (B6, at the age of 6-7 weeks). Samples were collected 4 or 5 weeks after MLN transfer and were subjected to ELISA and immunofluorescence and H\&E staining. 

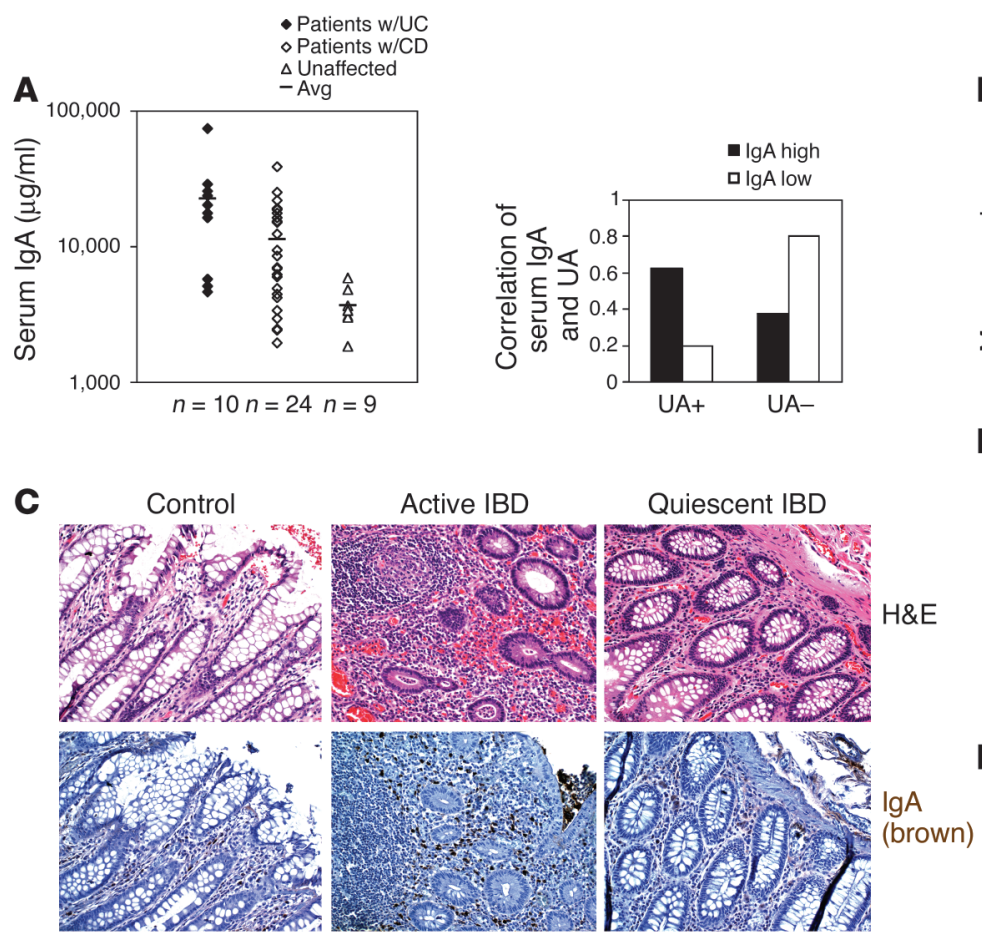
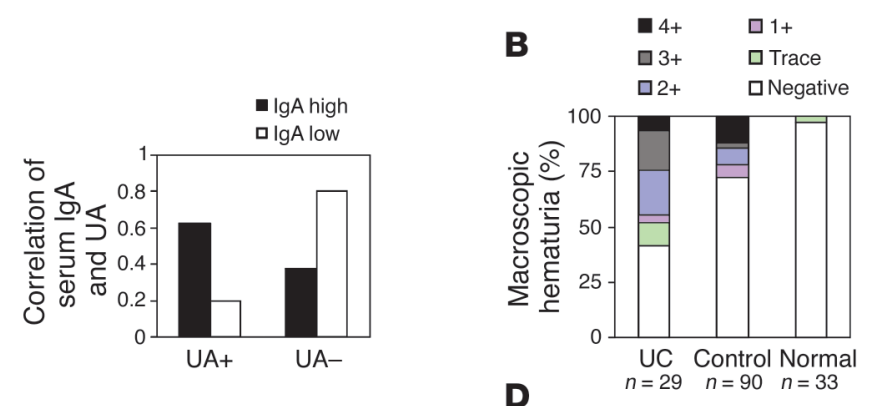

D

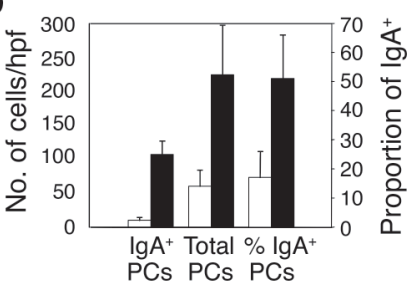

E

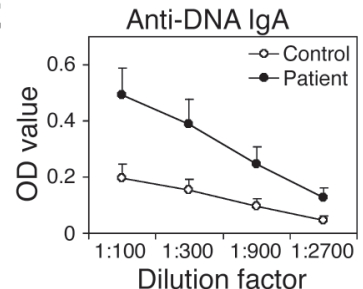

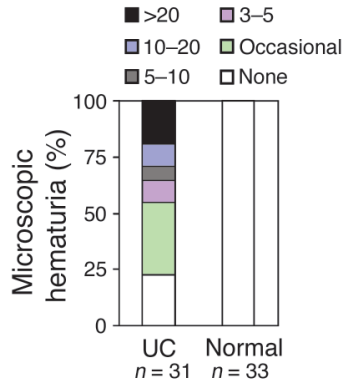

- Control patients - IBD patients

Figure 1

IBD patients show upregulated serum IgA levels and enhanced incidence of abnormal UAs. (A) Comparison of serum IgA in patents with ulcerative colitis (w/UC; $n=10$ ) or Crohn disease (w/CD; $n=24$ ) and unaffected control subjects (Unaffected; $n=9$ ) by ELISA illustrates the increase in serum IgA in IBD patients (left panel). Avg, average. Higher serum IgA levels correlate with a positive UA in IBD patients (right panel: UC, $n=9 ; \mathrm{CD}, n=17$ ). UA+, trace to 4+. (B) Macroscopic and microscopic UAs show the remarkable increase in hematuria in IBD patients (UC) compared with unselected patients (Control) or normal individuals (Normal) at the same hospital. (C) Immunohistochemical staining demonstrates increased lgA $\mathrm{ACs}^{+}$in the intestine of active IBD patients (Active IBD), while the quiescent IBD patients (Quiescent IBD) or control patients show much less positive staining for IgA ( $n=3$ per group). Severe intestinal inflammation was observed in active IBD patients but not in quiescent or control patients $(n=3 ; \mathrm{H} \& \mathrm{E})$. Magnification, $\times 200$. (D) PCs and IgA-PCs in LP were counted using a microscope. The numbers of PCs and IgA-PCs in active IBD patients were dramatically increased compared with those of controls. Cells in ten high-power fields (hpf's) were counted for each control or patient, and three controls or patients were analyzed. (E) Anti-DNA IgG and IgA antibodies were tested in controls $(n=5)$ and patients $(n=6)$. Data are means \pm SE of OD values.

\section{Results}

Dysregulated mucosal immunity in buman patients contributes to the IgAN pathogenesis. We hypothesized that a chronic increase in activated lymphocyte infiltration in the gut, such as in IBD, might serve as a potential mechanism to trigger dysregulation of IgA production. To test this hypothesis, we measured serum IgA in 34 human IBD patients and found that serum IgA was elevated in the majority of IBD patients (Figure 1A, left panel). Moreover, there was a strong correlation between elevated serum IgA and hematuria, a hallmark of IgAN, in the IBD patients (Figure 1A, right panel). Our data suggested that among the patients with serum IgA elevated above the mean levels for controls, $60 \%$ were UA (urine analysis) positive, while among the patients with serum IgA levels below the mean for controls, only $20 \%$ were UA positive (Figure $1 \mathrm{~A}$, right panel). To further validate our observations, we analyzed a larger cohort of IBD patients for macroscopic $(n=29)$ and microscopic $(n=31)$ hematuria. The incidence of hematuria was remarkably elevated in IBD patients compared with unselected control patients $(n=90)$ or normal individuals $(n=33)$ tested in the same hospital (Figure 1B), suggesting that there are renal function abnormalities in these IBD patients.
To determine if the elevated serum IgA in IBD patients reflects increased intestinal IgA production, we performed immunohistochemical analysis of resected tissue from IBD patients. Both the number and percentage of IgA $\mathrm{ICs}^{+}$were markedly increased in the gut of IBD patients relative to that of control patients (Figure 1D). Actively inflamed IBD tissues had significantly more IgA ${ }^{+}$PCs than did quiescent tissues in the same patients (Figure 1C). Moreover, the percentage of $\mathrm{IgA}^{+} \mathrm{PCs}$ in IBD patients was elevated much more than that of total PCs (Figure 1D). Thus, the data from human patients suggest a correlation between the dysregulated mucosal immune responses in active IBD patients and the increase in serum IgA and hematuria.

LIGHT-mediated hyperserum IgA and its requirement for LT $\beta R$. To dissect the mechanism for these interesting observations in human patients, we analyzed a transgenic line constitutively expressing LIGHT, a ligand for LT $\beta$ R, on the T cell lineage that spontaneously develops significant $T$ cell activation and intestinal inflammation (21). To systematically study the alternation at a molecular level, we initially studied transcription profiling by DNA microarrays that determined the mRNA expression of 11,000 murine genes and expressed sequence tags. The mRNA level of IgA was among 
the most dramatically increased transcripts, with a 35-fold increase in expression compared with that of age-matched WT controls (data not shown). This finding prompted us to examine the serum IgA levels in LIGHT Tg mice using ELISA. Serum IgA levels were remarkably elevated up to 30 - to 40 -fold in the Tg mice by the age of 6-8 months compared with those of WT controls (Figure 2A). Moreover, the elevation in serum IgA occurred in young Tg mice and actually preceded the histologically identifiable intestinal inflammation. At as early as 7 weeks of age, the level of serum IgA in $\mathrm{Tg}$ mice was tenfold higher than that in age-matched WT mice (Figure 2A). These results indicated a fundamental dysregulation of serum IgA production in LIGHT Tg mice. In addition, we found the extent of serum IgG elevation was much less significant than that of IgA in Tg mice (Figure 2B). Therefore, LIGHT Tg mice showed distinct hyperserum IgA, indicating a preferential dysregulation of IgA production.

To determine whether interaction between LIGHT and LT $\beta$ R is required for LIGHT-mediated serum IgA dysregulation, we performed an ELISA to test if the serum IgA levels in LIGHT Tg/LT $\beta R^{-/-}$ mice are elevated. In the absence of LT $\beta R$, the elevation of serum IgA disappeared completely even in the presence of the LIGHT transgene (Figure 2C). Moreover, LIGHT Tg/LT $\beta R^{-/-}$mice showed no gross abnormality in the intestine and no significant $\mathrm{T}$ cell-mediated inflammation was observed microscopically in the gut (data not shown). Thus, LT $\beta R$ is required for the resultant inflammation in the gut and hyperserum IgA in LIGHT Tg mice.

Increased glomerular deposition of IgA and complement $\mathrm{C} 3$ in LIGHT Tg mice. To determine whether elevated serum IgA leads to IgA deposition in the kidney, we performed immunofluorescence analyses of frozen sections of kidneys from LIGHT Tg and WT littermates. The glomeruli of WT mice were free of IgA immunofluorescence (Figure 3A), while LIGHT Tg mice showed a distinctive pattern of IgA immunofluorescence in the glomerular mesangium (Figure 3A). Many patients with IgAN also have glomerular accumulation of complement C3 $(12,13)$. Immunofluorescence analyses revealed glomerular deposition of complement C3 in the LIGHT Tg mice (Figure 3A); however, it was not detectable in the glomeruli of WT littermates (Figure 3A). In IgAN, IgG and/or IgM glomerular deposits are present in $60-83 \%$ and $22-66 \%$ of renal biopsies, respectively (30). Our data showed IgG and weak IgM deposition in the glomeruli of Tg mice, while no IgG- or IgM-specific fluorescence was detectable in those of WT mice (Figure 3A). This profile of predominant IgA and C3 glomerular deposition in the mesangium along with variable $\operatorname{IgG}$ and $\operatorname{IgM}$ staining is consistent with the pathological criteria for IgAN.

Histological examination of the kidney revealed glomerular and interstitial pathologic changes in Tg mice (Figure 3, E, G, H, and J). First, the Tg glomeruli appeared to have a striking change in the mesangium (Figure 3E). Compared with WT mice (Figure 3F), Tg mice had a wide acellular mesangium that was filled with a periodic acid-Schiff(PAS)-positive material (Figure 3G). In some instances the glomeruli were almost entirely sclerotic. Further investigation of the location of the deposits by electron microscopy revealed a diffuse electron-dense material in the mesangial cells of Tg mice (Figure 3C). The mesangial changes were either diffuse (Figure 3G) or focal (Figure $3 \mathrm{H}$ ), and we quantified these by light microscopy (Figure $3 \mathrm{~K}$ ). The $\mathrm{Tg}$ mice also demonstrated a patchy interstitial infiltrate without interstitial fibrosis (Figure 3J). The interstitial inflammation was not

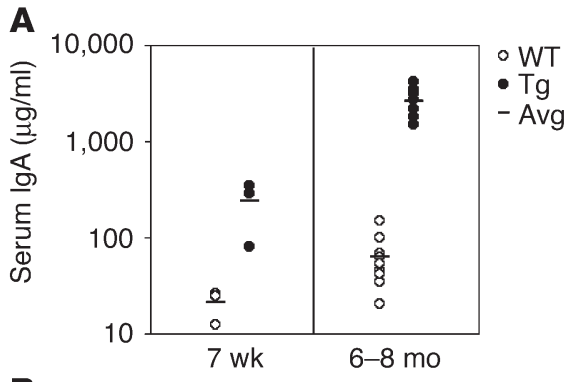

B
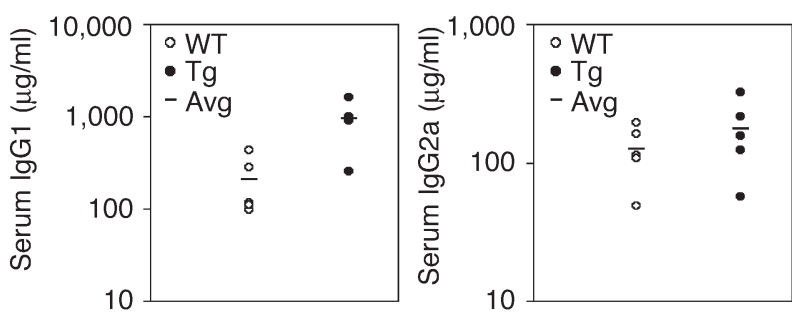

C

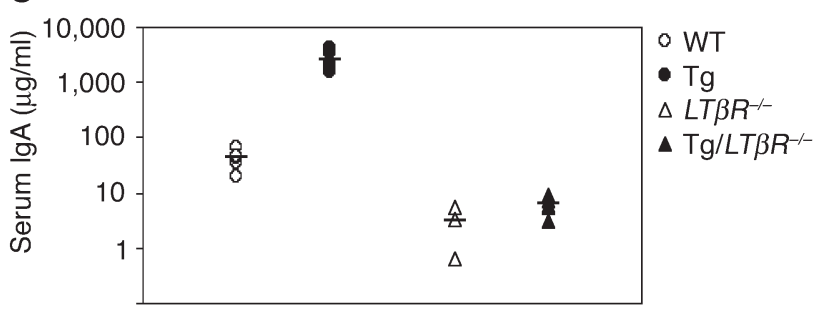

D

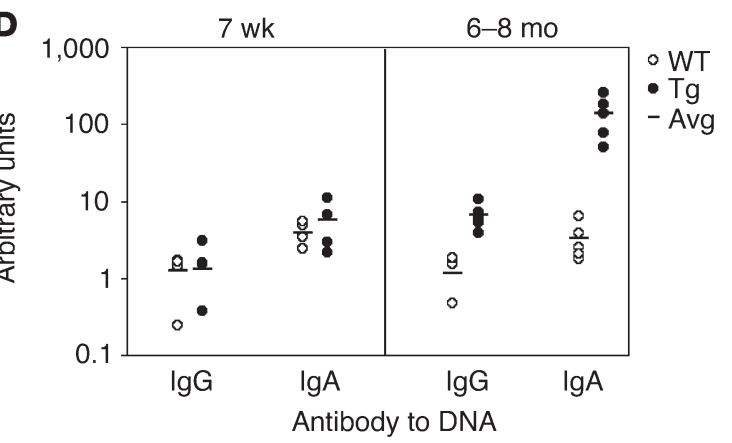

Figure 2

Elevated serum IgA in Tg mice. (A) Sera were collected from WT and Tg mice at the age of 6-8 months (right) or 7 weeks (left) and were subjected to IgA ELISA. Right, the level of serum IgA was dramatically enhanced in aged Tg mice $(n=10)$. Left, the level of serum IgA was increased up to tenfold in Tg mice ( $n=4$ per group). (B) Sera were collected from WT and Tg mice $6-8$ months of age and were subjected to IgG1 and IgG2a ELISA $(n=5)$. Open circles, WT; filled circles, Tg. (C) LIGHT Tg mice were crossed with $L T \beta R^{-/-}$mice. Sera were collected from $L T \beta R^{---}$and Tg/LT $\beta R^{-/-}$mice 6-8 months of age and IgA levels were determined by ELISA $(n=5)$. Hyperserum IgA was absent in $\mathrm{Tg} / L T \beta R^{-1-}$ mice compared with Tg mice. (D) Anti-DNA antibody was examined in WT and Tg mice ( $n=5$ per group). Anti-DNA IgG and anti-DNA IgA were both increased in aged Tg mice (6-8 months old), while there was no increase in young $\mathrm{Tg}$ mice (7 weeks old). 
A
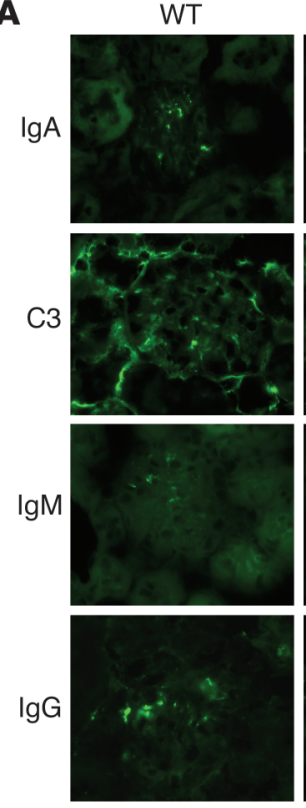

$\operatorname{Tg}$
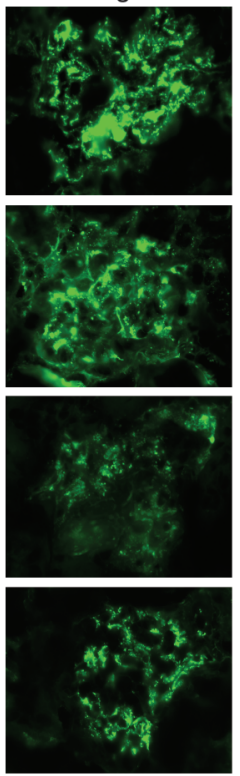

B
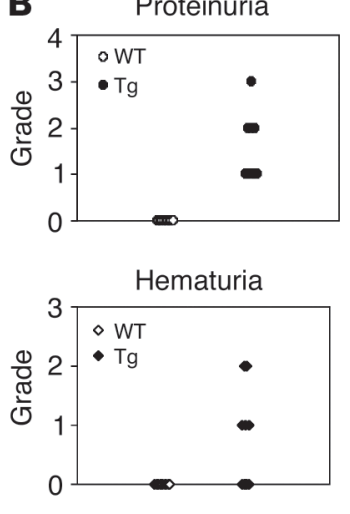

C

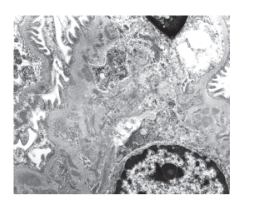

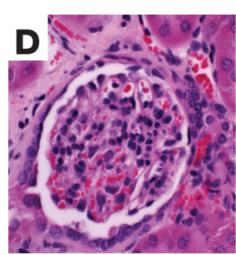
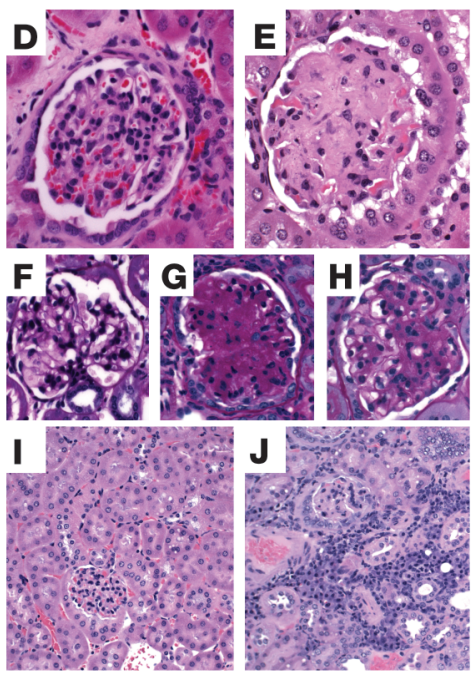

$\mathbf{K}$

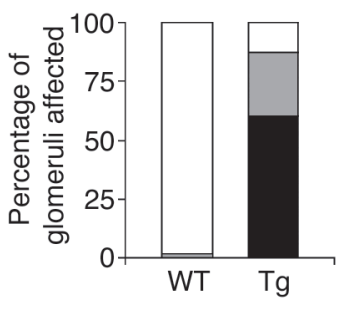

№ne

- Focal

- Diffuse

\section{Figure 3}

Dominant IgA deposition and abnormal UA in Tg mice. (A) Immunofluorescence staining of kidney sections of WT (left) and Tg (right) mice 6-8 months of age. Distinct IgA, C3, IgM, and IgG deposition were observed in aged Tg mice. Representative data are shown ( $n=3$ ). Magnification, $\times 630$. (B) Urine samples were subjected to strip UA. The incidence and severity of proteinuria $(n=10)$ and hematuria $(n=10)$ were higher in aged Tg mice (filled symbols) than in WT mice (open symbols). (C ) EM analysis of Tg glomeruli, showing mesangial deposition of immunoglobulin. (D and E) WT normal glomerulus (D) (H\&E; magnification, $\times 400)$ and Tg glomerulus with significant mesangial expansion with acellular eosinophilic material (E) (H\&E; magnification, $\times 400)$. ( $\mathbf{F}-\mathbf{H})$ WT normal glomerulus $(\mathbf{F})(\mathrm{PAS}$; magnification, $\times 400)$, and Tg glomerulus $(\mathbf{G}$ and $\mathbf{H})$ with diffuse $(\mathbf{G})$ and focal ( $\mathbf{H}$, right half of glomerulus) deposition of PAS-stainable material in the glomerular matrix. (I and $\mathbf{J})$ WT tubulointerstium with no significant inflammatory cellular infiltrate (I), and Tg tubulointerstium with patchy collections of inflammatory cellular infiltrates (J) (H\&E; magnification, $\times 200)$. (K) Semiquantitative assessment of the degree and severity of glomerular lesions in WT and Tg mice $(n=4$ mice per group).

present in the WT mice (Figure 3I). As in human IgAN, these pathologic changes were associated with hematuria and/or proteinuria. We examined the urine samples of WT and Tg mice and found significant spontaneous microscopic hematuria and proteinuria in aged $\mathrm{Tg}$ mice (Figure 3B). Taken together, the glomerular changes, the mesangial expansion with PAS-positive material, and the focal interstitial inflammation suggest that LIGHT Tg mice developed pathological features reminiscent of human IgAN.

Our previous study showed anti-DNA IgG antibody was elevated in $\mathrm{Tg}$ mice (21). We next tested further to see if anti-DNA IgA antibody was also present and found a dominant anti-DNA IgA response (Figure 2D). Due to the severe intestinal inflammation in Tg mice, it is likely that DNA was released from necrotic cells and exposed to the immune system, leading to the generation of antiDNA antibody. Therefore, we tested to see if anti-DNA antibody was present in young $\mathrm{Tg}$ mice that had elevated serum IgA (Figure 2A) but no evidence of intestinal inflammation (data not shown). The level of anti-DNA IgG and IgA in young Tg mice was very low and comparable to the levels in WT mice (Figure 2D). These results suggest that the anti-DNA antibody may be a consequence of self tissue damage, which occurs as the Tg mice age. Next, we tested IBD patients for anti-DNA antibody. Interestingly, IBD patients also showed a pattern of anti-DNA classes similar to that seen in the transgenic mice. Anti-DNA IgA was significantly elevated in IBD patients, while anti-DNA IgG was only slightly increased (Figure 1E). Thus, our data suggest that our animal model and human IBD patients may share similarities in pathogenic mechanisms.
Increase in IgA+ PCs in PPs and colon. IgA-PCs have been shown to be derived from the antigen-specific IgA-committed B cells in PPs (5). Therefore, we next examined the B cell populations in PPs and detected a much higher percentage of B220- $\mathrm{IgA}^{+}$and B220 $0^{+} \mathrm{IgA}^{+} \mathrm{lym}-$ phocytes in PPs (Figure 4A). The former population, B220- $\mathrm{IgA}^{+}$cells, has been proven to be IgA-PCs (31). As shown in Figure 4A, IgA+ PCs constituted about $1 \%$ of PP lymphocytes in WT littermates, whereas about $7 \%$ of cells were $\operatorname{IgA}^{+}$in PPs of LIGHT Tg mice. B220+ $\operatorname{IgA}{ }^{+}$ cells were also increased twofold (Figure 4A). Recent studies have shown that this particular population has a distinct phenotype and is at transitional stage, with newly completed class switching, and will undergo terminal differentiation to PCs (6).

Serum IgA in rodents has been suggested to originate in part from pIgA produced in the intestine (32). Therefore, we examined the intestine with immunohistochemical staining and found that there were increased numbers of $\operatorname{IgA}^{+}$PCs that massively infiltrated the LP of Tg mice (Figure 4B, top panel). The number of $\mathrm{IgA}^{+} \mathrm{PCs}$ was dramatically higher in $\mathrm{Tg}$ mice than in WT controls (Figure 4B, top panel). There were many lymphoid follicle-like structures infiltrating the LP of the Tg colon. We speculate that activation of LT $\beta R$ in gut stromal tissue by LIGHT may upregulate adhesion molecules for recruiting inflammatory cells and $\operatorname{IgA}$ precursors. LT $\beta R$ signaling is important for MAdCAM-1 expression (33), a critical adhesion molecule for the migration of IgA precursor into in the gut (34). Consistent with the findings in refs. 33 and 34, there was increased MAdCAM-1 expression in the colon of Tg mice (Figure 4B, bottom panel). These findings indi- 

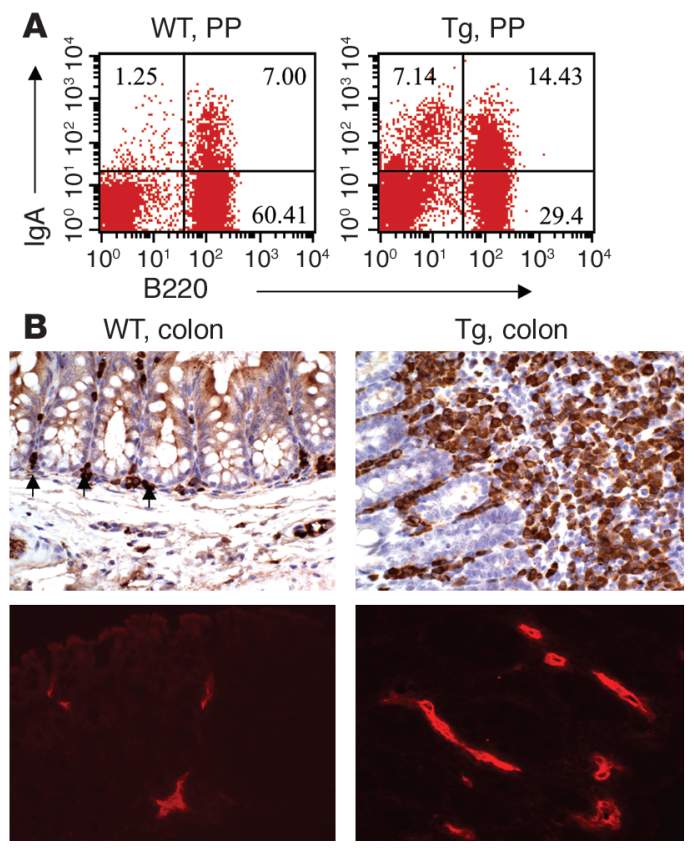

$\lg A$
$(\times 400)$
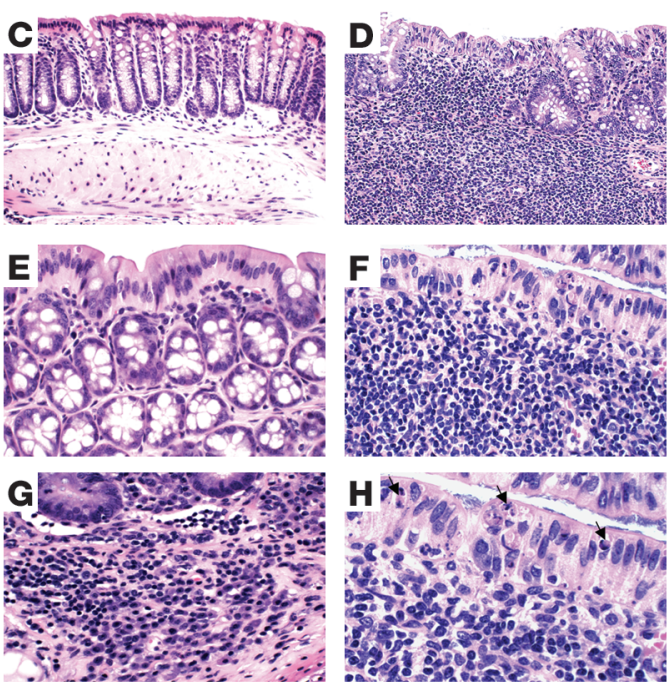

\section{Figure 4}

Increase in $\lg \mathrm{A}^{+}$cells in PPs and colon. (A) The frequency of $\lg \mathrm{A}^{+}$and B220+ $\lg \mathrm{A}^{+}$cells in PPs. Representative FACS profiles of IgA and B220 staining of WT (left) and Tg (right) PP cells from mice 6-8 months of age are shown. $\lg \mathrm{A}^{+}$and $\mathrm{B}_{22} \mathrm{O}^{+} \lg \mathrm{A}^{+}$cells were increased in PPs of Tg mice $(n=4)$. (B) Immunohistochemical staining of intestine for $\lg A^{+}$PCs (indicated by arrows) in WT and Tg mice (top panels). Magnification, $\times 400$. Upregulation of MAdCAM-1 expression in Tg intestine (bottom panels). Intestine sections were stained with anti-MAdCAM-1 and subjected to fluorescence microscopy. Representative pictures are shown for WT and Tg mice $6-8$ months of age $(n=3)$. Magnification, $\times 200$. (C-H) H\&E staining for WT (C and $\mathbf{E})$ or $\operatorname{Tg}(\mathbf{D}, \mathbf{F}-\mathbf{H})$ intestine, showing a dramatic infiltration of inflammatory cells in $\mathrm{Tg}$ mice. In Tg intestine, there were increases in PCs (G) and apoptosis of epithelial cells (H, arrow). Magnifications: $\times 200(\mathbf{C}$ and D), $\times 400(\mathbf{E}-\mathbf{G})$, and $\times 1,000(\mathbf{H})$.

cate that overexpression of LIGHT may signal LT $\beta$ R to create local environment for increased influx of $\mathrm{T}$ cells and IgA-producing B cells into the intestines of $\mathrm{Tg}$ mice.

Impairment of the epithelial layer and defective IgA transportation in the intestine. LIGHT Tg mice developed intestinal inflammation with markedly increased LP neutrophils, lymphocytes, PCs, and focal epithelial cell damage (Figure 4, D and F-H). The epithelial cell damage was associated with intraepithelial neutrophils, lymphocytes, and occasional crypt abscesses (Figure 4F). Increased apoptosis of gut epithelial cells was also observed in $\mathrm{Tg}$ mice (Figure $4 \mathrm{H}$, arrows). In addition to this mucosal damage, the intestinal wall was diffusely thickened in LIGHT Tg mice (Figure 4D). Under higher magnification, many of infiltrating cells displayed a typical PC morphology, including an eccentric nucleus, low nuclear-tocytoplasmic ratio, a dark basophilic cytoplasm, and a pale perinuclear compartment (Figure 4G). In addition, immunofluorescence microscopy showed that many of the LP leukocytes were T cells and granulocytes (data not shown). These findings suggest that upregulation of LIGHT on T cells leads to activation of T cells, mucosal inflammatory infiltrates, epithelial damage, and intestinal wall thickening. These pathogenic features overlap with those seen in the intestinal mucosa of human IBD patients. An independent study also showed intestinal inflammation in CD2LIGHT Tg mice (35).

Next, we wondered whether a chronic inflammatory condition might be sufficient to produce some deterioration in the function of intestinal epithelial cells, such as transport of IgA into mucosal secretions. Therefore, we determined IgA levels in fecal extracts from WT and Tg mice. The IgA level in the secretions of aged
LIGHT Tg was significantly decreased (Figure 5A) and correlated well with the severity of the intestinal inflammation (Figure 4). Thus, we have demonstrated downregulation of IgA excretion into the intestinal lumens of Tg mice, presumably caused by impairment of intestinal epithelial function.

Increased polymeric serum IgA in Tg mice. The intestinal source of IgA usually exists in a polymeric form, which is prone to deposition in the glomerular mesangium. Given the increase in $\operatorname{IgA}^{+} \mathrm{PCs}$, the impairment of the gut epithelium, and the reduction of IgA in secretion, we speculated that PIgA might contribute to hyperserum IgA syndrome. To investigate the origin of the pathogenic IgA for mesangial deposition, we tested serum IgA in LIGHT Tg mice for its monomeric versus polymeric nature by FPLC. The concentration of IgA in each fraction after FPLC was confirmed by anti-IgA ELISA. Whereas pIgA has a molecular weight of more than $350 \mathrm{kDa}$, that of monomeric IgA (mIgA) is about $160 \mathrm{kDa}$. Our results showed that pIgA is predominant in the serum of LIGHT Tg mice in contrast to that of WT controls (Figure 5B), and the ratio of pIgA to mIgA was significantly enhanced in the serum of Tg mice. These results suggested that the increased IgA in the serum of LIGHT Tg mice was derived from mucosal tissues.

To test if there is a catabolism defect in the serum pIgA in LIGHT $\mathrm{Tg}$ mice, we purified PIgA from serum of Igh ${ }^{\mathrm{a}}$ mice by FPLC and administered $\mathrm{pIgA}$ a intravenously to WT or Tg mice. After 2 weeks, serum $\operatorname{Ig} \mathrm{A}^{\mathrm{a}}$ was monitored by ELISA with antibody against $\operatorname{Ig} \mathrm{A}^{\mathrm{a}}$ allele (29). The level of $\mathrm{pIgA}^{\mathrm{a}}$ in serum was significantly higher in $\mathrm{Tg}$ recipients than WT recipients (Figure $5 \mathrm{C}$ ). These data suggest that the half-life of $\mathrm{pIgA}^{\mathrm{a}}$ is longer in $\mathrm{Tg}$ mice and that $\mathrm{Tg}$ mice may have additional defects in clearing a given amount of $\mathrm{pIgA}^{\text {a }}$ from the cir- 


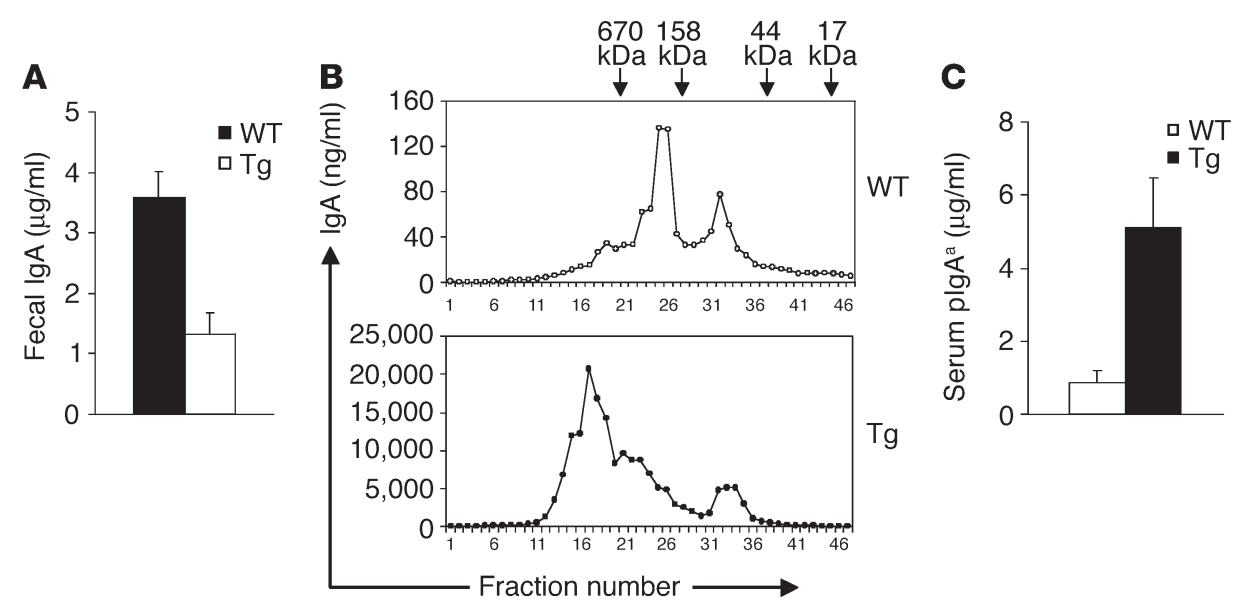

Figure 5

Defective IgA transportation and polymeric nature of serum IgA. (A) Feces of mice 8-10 months of age were collected, and IgA levels in the fecal extracts were determined by ELISA. Fecal IgA levels in Tg mice decreased significantly. Data are mean values \pm SE from five mice per group. Student's $t$ test was used to compare the values between the WT and Tg groups: $P<0.05$. (B) FPLC fraction profiles of IgA in sera. Gelfiltration molecular mass marker for protein was used. IgA levels in fractioned samples of sera were determined by ELISA. plgA is predominant in sera of Tg mice compared with WT mice 6-8 months old. (C) After plgA was purified from serum of Igha mice, it was administered i.v. to WT or Tg mice 8-10 months of age $(n=5)$. The level of plgA ${ }^{a}$ was tested by ELISA against lgA ${ }^{a}$. Tg recipients showed much higher levels of plgA ${ }^{a}$.

culation (Figure 5C). Taken together, our results have demonstrated that the chronic inflammation in the intestinal environment of $\mathrm{Tg}$ mice eventually led to the increase in gut-derived serum pIgA.

The recapitulation of $T$ cell-mediated intestinal inflammation and IgAN. To determine whether LIGHT-mediated lymphocyte activation constitutes a crucial step for the development of intestinal inflammation and IgA deposits, we transferred LN cells from WT or Tg mice into $R A G-1^{-/-}$mice and monitored the disease progression. $R A G-1^{-/-}$mice reconstituted with $\mathrm{Tg} \mathrm{LN}$ cells (Tg recipients) spontaneously developed colitis by $4-5$ weeks (Figure $6 \mathrm{~A}$ ). $R A G-1^{-/-}$ mice receiving Tg LN cells displayed marked LP infiltrates, promi- nent crypt abscesses, and crypt architectural distortion (Figure 6A). Therefore, our data indicated that intestinal infiltration could be transferred by $\mathrm{T}$ cells with upregulated LIGHT expression, further demonstrating the pathogenicity of these lymphocytes. Next we investigated if LN cells derived from Tg mice could induce hyperserum IgA. The serum IgA level was substantially elevated in $\mathrm{Tg}$ recipients, as determined by ELISA 4 weeks after adoptive transfer (Figure 6B). In addition, the serum IgA level was further augmented by 5 weeks in Tg recipients, compared with the results at 4 weeks (Figure 6B). Consistent with our hypothesis, such $\mathrm{T}$ cell-mediated intestinal inflammation led to the IgA deposition in the kidneys of $\mathrm{Tg}$ recipients (Figure 6C, IgA). Moreover, the glomeruli of $\mathrm{Tg}$ recipients were partially or completely obliterated, and increased mesangial matrix deposition was evident in the glomeruli of $\mathrm{Tg}$ recipients (Figure $6 \mathrm{C}, \mathrm{H} \& \mathrm{E})$. Therefore, the etiological cascade of IgAN was recapitulated in our adoptive transfer model and the unique features of LIGHT were essential for triggering the occurrence of both intestinal inflammation and the establishment of a proper environment for IgA production in the intestine.

The connection between gut-derived byperserum IgA and IgAN using a non-Tg model. Next, we sought to determine if the etiological cascade is unique to our Tg mice or is a general mechanism. Therefore, another experimental model for $\mathrm{T}$ cell-mediated gut inflammation was used. After transfer of BM and splenocytes of C57BL/6 (B6) donors into $\mathrm{LP} / \mathrm{J}$ recipients $(\mathrm{B} 6 \rightarrow \mathrm{LP} / \mathrm{J})$, donor $\mathrm{T}$ cells were activat-
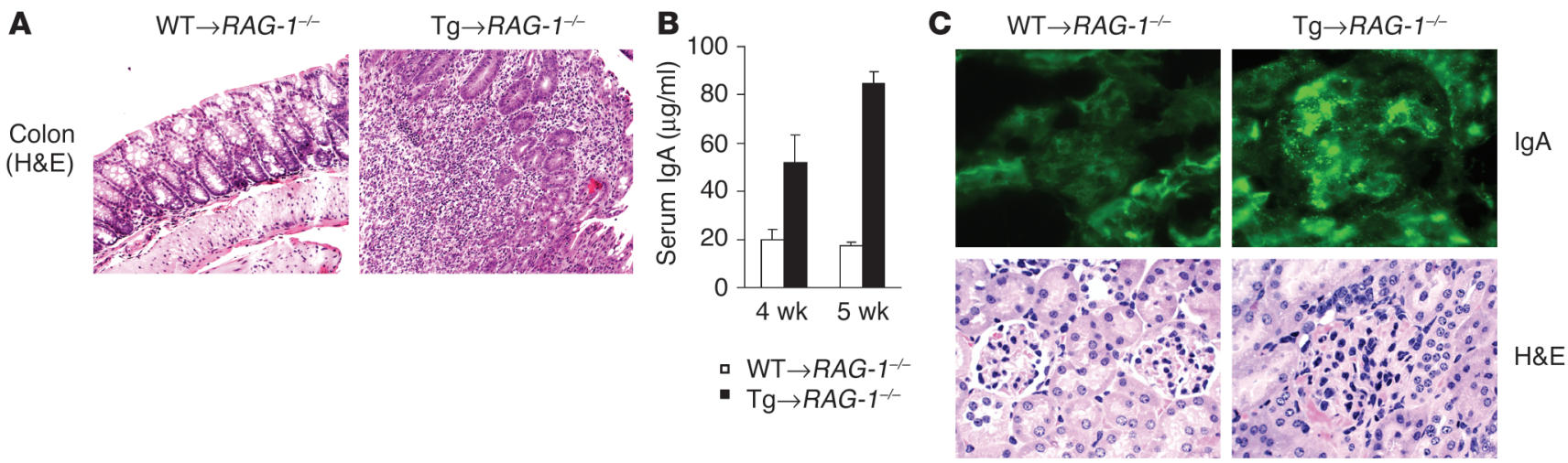

Figure 6

Recapitulation of IBD and IgAN in RAG-1-/- mice with adoptively transferred Tg LN cells. (A-C) LN cells from WT or Tg mice 2-3 months of age were transferred into $R A G-1^{-1-}$ mice $6-7$ weeks old $\left(6 \times 10^{6}\right.$ cells/mouse; $\left.n=4\right)$. (A) Disease progression was monitored. More severe intestinal inflammation was seen in Tg recipients (right panel) than in WT recipients (left panel). (B) Serum IgA was measured by ELISA 4 or 5 weeks after transfer. Serum IgA in Tg recipients (black bars) was elevated compared with that of WT recipients (white bars). (C) Top, kidney sections from WT recipients (left) and Tg recipients (right) were subjected to IgA immunofluorescence staining. Magnification, $\times 630$. Bottom, H\&E staining of kidney sections from WT (left) or Tg (right) recipients shows the obliterated glomeruli and glomerular mesangial matrix deposition in Tg recipients. 

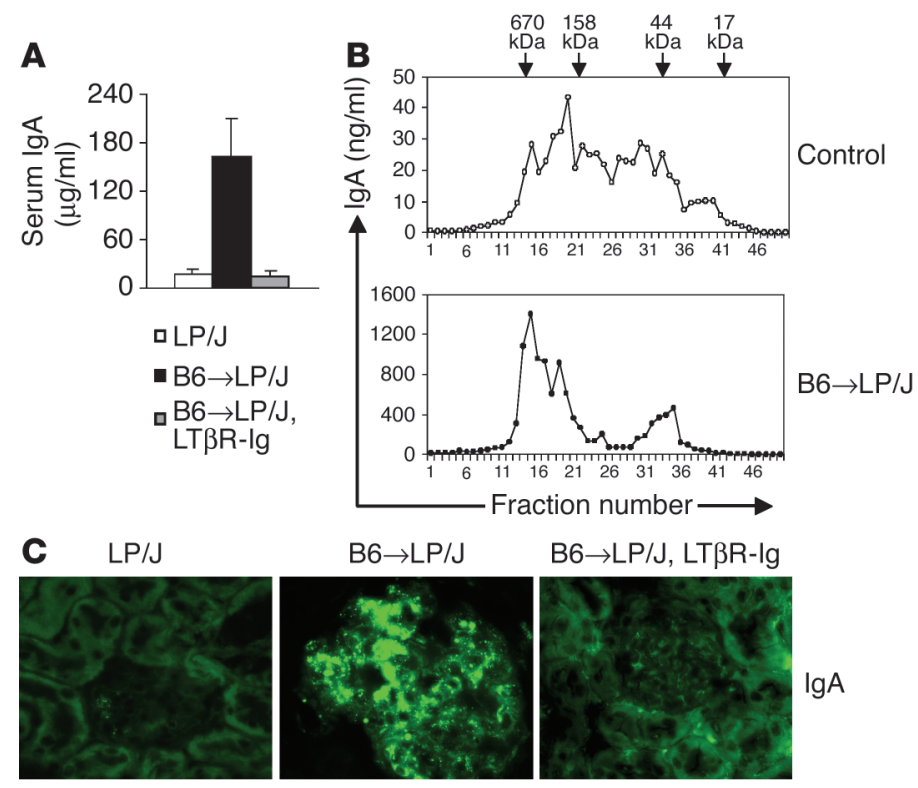

$B 6 \rightarrow$ LP/J, LT $\beta R-\lg$

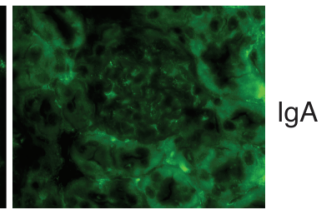

Figure 7

The connection between hyperserum $\lg \mathrm{A}$ and $\lg \mathrm{A}$ deposition in an activated $\mathrm{T}$ cell-mediated model. $(\mathbf{A}-\mathbf{C})$ BM cells $\left(2 \times 10^{6}\right)$ and splenocytes $\left(5 \times 10^{7}\right)$ from B6 donors were transferred i.v. into MiHC-incompatible LP/J recipients. Serum was collected 20 days after transfer from control mice (LP/J), B6 $\rightarrow$ LP/J mice, and B6 $\rightarrow$ LP/J mice treated with LT $\beta R-\lg (n=3$ per group). (A) IgA ELISA was performed. (B) FPLC fraction profiles of IgA in sera of the control and B6 $\rightarrow \mathrm{LP} / \mathrm{J}$ groups. Gel-filtration molecular mass marker for protein was used. IgA levels in fractioned samples from sera were determined by ELISA. plgA is predominant in sera of the B6 $\rightarrow$ LP/J group compared with control. (C) Kidney sections were subjected to immunofluorescence staining for IgA. Representative data are shown. Magnification, $\times 630$.

ed by minor histocompatibility (MiHC) antigens, and LP/J recipients gradually developed extensive $\mathrm{T}$ cell infiltration in the intestines and GVHD phenotypes (date not shown). Interestingly, the level of serum IgA was significantly enhanced in mice developing intestinal inflammation $(\mathrm{B} 6 \rightarrow \mathrm{LP} / \mathrm{J})$ compared with control LP/J mice (Figure 7A). The majority of elevated serum IgA was polymeric, as shown by FPLC, in the B $6 \rightarrow \mathrm{LP} / \mathrm{J}$ group, suggesting an intestinal origin (Figure 7B). IgA deposition in glomeruli was also assessed by immunofluorescence staining. Kidneys from $\mathrm{B} 6 \rightarrow \mathrm{LP} / \mathrm{J}$ mice showed massive mesangial IgA deposition (Figure 7C, B6 $\rightarrow \mathrm{LP} / \mathrm{J}$ ); in contrast, no IgA deposition was observed in the glomeruli of LP/J mice (Figure $7 \mathrm{C}, \mathrm{LP} / \mathrm{J}$ ). In addition, mesangial matrix expansion was detected in the kidneys of $\mathrm{B} 6 \rightarrow \mathrm{LP} / \mathrm{J}$ mice (Figure $7 \mathrm{C}, \mathrm{B} 6 \rightarrow \mathrm{LP} / \mathrm{J}$ ). Codeposition of IgG was also observed in some glomeruli of $\mathrm{B} 6 \rightarrow$ $\mathrm{LP} / \mathrm{J}$ mice; however, the percentage of glomeruli affected by IgG deposition was less than that of IgA deposition (data not shown).

To determine whether upregulation of LIGHT could be involved in pathogenesis and to develop new treatments, we administered LT $\beta$ R-Ig, a soluble receptor fusion protein for LIGHT, to LP/J recipients. Impressively, the level of serum IgA in the LT $\beta$ R-Ig-treated group was reduced to that of normal LP/J mice (Figure 7A), suggesting that upregulation of serum pIgA may be attributed to the engagement of LT $\beta$ R by its ligand LIGHT. The accumulation of mesangial IgA was substantially decreased by treatment with LT $\beta$ R-Ig (Figure 7C, B6 $\rightarrow$ LP/J, LT $\beta$ R-Ig). Overall, the pathological features were recapitulated in the $\mathrm{B} 6 \rightarrow \mathrm{LP} / \mathrm{J}$ transfer model, further vali- dating the observation in LIGHT Tg mice that disturbed mucosal immune responses can lead to overproduction of pIgA and IgA deposition in the glomeruli.

\section{Discussion}

Using a LIGHT Tg model, we have demonstrated here an etiological cascade for IgAN that was initiated by abnormally activated $\mathrm{T}$ cells, followed by severe intestinal inflammation, resulting in profound dysregulation of serum IgA levels via overproduction and impaired transportation. The combination of these abnormalities led to dramatically elevated serum IgA, predominantly pIgA due to its intestinal origin, which eventually caused glomerular IgA deposition. Our study highlights the central role of $\mathrm{T}$ cells and mucosal immune responses in IgAN development. Importantly, the IgAN phenotype was found to be dependent on LT $\beta$ R signaling in our models. Moreover, we have developed a potential treatment that can ameliorate IgAN.

A striking increase in PIgA was detected in the serum of aged Tg mice, which further indicates that IgA-PCs in the intestine are probably the major source of the increased serum $\operatorname{IgA}$, due to the predominant production of PIgA in the gut and gut-associated lymphoid tissue (GALT) (32). The pathogenic role of pIgA has been well established in IgAN. Owing to its multivalent properties, pIgA can form immune complexes more efficiently, and these macromolecular complexes are subsequently entrapped in the kidney because of their size $(>500 \mathrm{kDa})$ or via specific binding to potential IgA receptors on mesangial cells in the kidney (36). The level of serum IgA is considered to be dependent not only on production and catabolism but also on excretion into the gut lumen via the intestinal epithelium and biliary tract (37). The downregulation of IgA excretion into the intestinal lumens of $\mathrm{Tg}$ mice (Figure 5) indicated the compromised function of the intestinal epithelial cells. Although whether IgA deposition in the kidneys of IgAN patients originates from mucosa or BM still remains a point of controversy (38-40), our model seems to indicate the former possibility. Our data from human IBD patients also suggest that a dysregulated mucosal immune response led to the increased serum IgA and IgA $\mathrm{I}^{+}$-PCs in the colon.

Increased $\operatorname{IgA}$ production and subsequent deposition play a dominant role in the glomerular damage; however, we cannot rule out the possibility that antibodies against DNA may contribute to the glomerular abnormality. First, LIGHT Tg mice overall have a dominant IgA response, whereas in MRL/lpr mice, a well-established model for systemic lupus erythematosus-like autoimmune syndrome, the IgG response appears to be dominant (data not shown). Furthermore, anti-DNA IgG in Tg mice is only about four- to fivefold higher than that in WT mice (21). However, the level of anti-DNA IgG in MRL/lpr mice was about 50- to 100-fold higher than that of age-matched LIGHT Tg mice (data not shown). Finally, we also tested anti-DNA IgA production in $\mathrm{Tg}$ mice. Again, the dominant class of anti-DNA antibody was IgA in LIGHT Tg mice. Interestingly, young Tg mice had very low antibodies against DNA, although they did have increased serum IgA (Figure 2). These results suggest that the increase in total IgA occurs earlier and independently of antibodies against DNA and that antibodies against DNA may be a consequence of self tissue damage in aged $\mathrm{Tg}$ mice. As the intestinal environment favors IgA class switching (6), a dominant anti-DNA IgA response was 
observed in $\mathrm{Tg}$ mice. Taken together, our results indicate increased pIgA plays a dominant role in renal damage and autoantibodies may serve as a contributing factor.

To discern between a LIGHT-specific perturbation in the gut leading to an increase in serum IgA versus hyperserum IgA resulting simply as a consequence of an inflamed gut environment, we examined serum IgA levels in young Tg mice (7 weeks old) and found that young Tg mice had serum IgA tenfold higher than that of WT mice (Figure 2). These young Tg mice had no weight loss or diarrhea, their intestines were grossly normal, and no evident inflammation was observed (data not shown). Our data suggest that the increased IgA at a young age may be caused primarily by overproduction of IgA in the gut, while subsequent intestinal inflammation may cause further overproduction and impaired transport of IgA into lumen. The compound effects of all these elements eventually lead to the dramatically increased serum pIgA in aged $\mathrm{Tg}$ mice. Such phenotypes are probably difficult to reproduce exactly in the adoptive transfer model.

Our proposed mechanism of IgAN pathogenesis not only is applicable to the LIGHT Tg system but also is supported by results from another experimental model (Figure 7). Administration of LT $\beta$ RIg, a soluble receptor for LIGHT, significantly reduced the accumulation of glomerular IgA in LP/J recipients, suggesting involvement of LIGHT and disturbance of the LT $\beta$ R pathway as a common mechanism for the pathogenesis IgAN. Previous studies have shown that treatment with LT $\beta$ R-Ig can block the development of GVHD (20) and colitis (41) and that GALT is the major site initiating the GVHD responses (42). Therefore, an alternative explanation for the effects of LT $\beta$ R-Ig on IgAN may be reduced GVHD or colitis mediated by LT $\beta$ R-IG treatment, and specific involvement of gut IgA production needs to be further elucidated. In $60 \%$ of IgAN patients that undergo renal transplantation, the disease recurs in the transplanted kidney (43). Our results suggest an important application for LT $\beta$ R-Ig in the treatment of IgAN.

We would like to point out the complexity and uniqueness of our model. Not all intestinal inflammation or colitis is associated with an increase in serum IgA; for example, our preliminary data showed that IL-2 $2^{-/-}$mice had no increase in serum IgA. Moreover, the severity of the colitis is not always associated with the extent of the increase in serum IgA. In our adoptive transfer model, the increase in serum IgA in the $\mathrm{Tg}$ recipients was not as dramatic as that of the parental line, although the $\mathrm{Tg}$ recipients did develop very severe intestinal inflammation. Finally, low levels of dextran sulfate sodium were reported to enhance serum IgA, while high levels decreased it (44). The absence of high serum IgA with colitis in some models suggested that LIGHT itself was the critical component in our models and that the unique features of LIGHT are crucial to the disease pathogenesis. Our previous study showed that LT $\beta R$ in the intestine is required for IgA production and for the recruitment of IgA precursors (16), and the LIGHT transgene indeed signals LT $\beta \mathrm{R}$ in vivo (24). Thus, LIGHT probably can interact with LT $\beta R$ on the stromal cells in the gut and promotes IgA production and recruitment of IgA precursors. Our data also suggest that LIGHT may cause an increase in serum IgA in the absence of severe intestinal inflammation in young $\mathrm{Tg}$ mice. Due to the costimulatory and proinflammatory activity of LIGHT $(20,21,35)$, intestinal inflammation developed in the aged $\mathrm{Tg}$ mice and such inflammation further contributed to the increase in serum PIgA. In addition, interaction between LIGHT and LT $\beta$ R can promote upregulation of adhesion molecules and chemokines, which may also recruit more inflammatory cells into the intestine (45). Thus, treatment with LT $\beta$ R-Ig in the MiHC mismatch model probably can block both IgA production and inflammation in the intestine.

LIGHT, as a key molecule, is critical for the generation of activated $\mathrm{T}$ cells homing to the gut, leading to severe inflammation in the gut and IgA deposition in the kidney. The results of our study here show that $\mathrm{T}$ cell-mediated intestinal abnormality in certain cases can lead to the progression of IgAN. Our model may be related more to secondary IgAN. The distinction between the primary and secondary forms of IgAN is that most patients with primary ("idiopathic") IgAN do not produce autoantibodies against DNA or display evidence of florid intestinal inflammation, whereas secondary IgAN is known to occur in association with IBD, celiac disease, and other inflammatory conditions (25-28). A better understanding of such mechanisms may allow us to make proper diagnoses and to develop therapeutic approaches to human IgAN in the future.

\section{Acknowledgments}

This research was supported by grants from the National Institutes of Health (HD-37104 and DK-58897), the Juvenile Diabetes Foundation International (1-2000-875), and Biogen. J. Wang and Y. Sun are recipients of NIH training grant 5T32 HL07237. Immunofluorescence images were obtained and processed at the Digital Light Microscopy Facility at the University of Chicago. Immunohistochemical staining for human samples were performed by the histology laboratory of the Department of Pathology at the University of Chicago. Immunohistochemical staining of IgA for murine samples was performed by the Immunology Applications Core Facility at the University of Chicago.

Received for publication September 22, 2003, and accepted in revised form January 6, 2004.

Address correspondence to: Yang-Xin Fu, Department of Pathology, MC6027, The University of Chicago, 5841 South Maryland Avenue, Chicago, Illinois 60637, USA. Phone: (773) 702-0929; Fax: (773) 834-8940; E-mail:yfu@midway.uchicago.edu.

\footnotetext{
1. Gardby, E., Lane, P., and Lycke, N.Y. 1998. Requirements for B7-CD28 costimulation in mucosal IgA responses: paradoxes observed in CTLA4-H $\gamma 1$ transgenic mice. J. Immunol. 161:49-59.

2. Vajdy, M., Kosco-Vilbois, M.H., Kopf, M., Kohler, G., and Lycke, N. 1995. Impaired mucosal immune responses in interleukin 4-targeted mice. J. Exp. Med. 181:41-53.

3. Hornquist, C.E., Ekman, L., Grdic, K.D., Schon, K., and Lycke, N.Y. 1995. Paradoxical IgA immunity in CD4-deficient mice. Lack of cholera toxin-specific protective immunity despite normal gut mucosal IgA differentiation. J. Immunol. 155:2877-2887.

4. Lycke, N., Eriksen, L., and Holmgren, J. 1987. Protection against cholera toxin after oral immuniza-
}

tion is thymus-dependent and associated with intestinal production of neutralizing IgA antitoxin. Scand. J. Immunol. 25:413-419.

5. Craig, S.W., and Cebra, J.J. 1971. Peyer's patches: an enriched source of precursors for IgA-producing immunocytes in the rabbit. J. Exp. Med. 134:188-200.

6. Fagarasan, S., Kinoshita, K., Muramatsu, M., Ikuta, K., and Honjo, T. 2001. In situ class switching and differentiation to IgA-producing cells in the gut lamina propria. Nature. 413:639-643

7. Husband, A.J., and Gowans, J.L. 1978. The origin and antigen-dependent distribution of IgA-containing cells in the intestine. J. Exp. Med. 148:1146-1160.

8. Brandtzaeg, P. 1981. Transport models for secretory IgA and secretory IgM. Clin. Exp. Immunol. 44:221-232.
9. Berger, J., and Hinglais, N. 1968. [Intercapillary deposits of IgA-IgG]. J. Urol. Nephrol. (Paris). 74:694-695.

10. Cagnoli, L., et al. 1985. B and T cell abnormalities in patients with primary IgA nephropathy. Kidney Int. 28:646-651.

11. Woodroffe, A.J., et al. 1980. Immunologic studies in IgA nephropathy. Kidney Int. 18:366-374.

12. van Es, L.A., van den Wall Bake, A.W., Valentijn, R.M., and Daha, M.R. 1988. Composition of IgA-containing circulating immune complexes in IgA nephropathy. Am. J. Kidney Dis. 12:397-401.

13. Rifai, A., Small, P.A., Jr., Teague, P.O., and Ayoub, E.M. 1979. Experimental IgA nephropathy. J. Exp. Med. 150:1161-1173. 
14. Harper, S.J., and Feehally, J. 1993. The pathogenic role of immunoglobulin A polymers in immunoglobulin A nephropathy. Nephron. 65:337-345.

15. Nagura, H. 1989. IgA nephropathy and mucosal immune system. Tokai J. Exp. Clin. Med. 14:1-4.

16. Kang, H.S., et al. 2002. Signaling via LT $\beta R$ on the lamina propria stromal cells of the gut is required for IgA production. Nat. Immunol. 3:576-582.

17. Futterer, A., Mink, K., Luz, A., Kosco-Vilbois, M.H., and Pfeffer, K. 1998. The lymphotoxin $\beta$ receptor controls organogenesis and affinity maturation in peripheral lymphoid tissues. Immunity. 9:59-70.

18. Mauri, D.N., et al. 1998. LIGHT, a new member of the TNF superfamily, and lymphotoxin $\alpha$ are ligands for herpesvirus entry mediator. Immunity. 8:21-30.

19. Scheu, S., et al. 2002. Targeted disruption of LIGHT causes defects in costimulatory $\mathrm{T}$ cell activation and reveals cooperation with lymphotoxin $\beta$ in mesenteric lymph node genesis. J. Exp. Med. 195:1613-1624.

20. Tamada, K., et al. 2000. Modulation of T-cell-mediated immunity in tumor and graft-versus-host disease models through the LIGHT co-stimulatory pathway. Nat. Med. 6:283-289.

21. Wang, J., et al. 2001. The regulation of T cell homeostasis and autoimmunity by $\mathrm{T}$ cell-derived LIGHT. J. Clin. Invest. 108:1771-1780. doi:10.1172/ JCI200113827.

22. Ye, Q., et al. 2002. Modulation of LIGHT-HVEM costimulation prolongs cardiac allograft survival. J. Exp. Med. 195:795-800.

23. Wu, Q., et al. 2001. Reversal of spontaneous autoimmune insulitis in nonobese diabetic mice by soluble lymphotoxin receptor. J. Exp. Med. 193:1327-1332.

24. Wang, J., et al. 2002. The complementation of lymphotoxin deficiency with LIGHT, a newly discovered TNF family member, for the restoration of secondary lymphoid structure and function. Eur. J. Immunol.32:1969-1979.
25. Emancipator, S.N. 1990. Immunoregulatory factors in the pathogenesis of IgA nephropathy. Kidney Int. 38:1216-1229.

26. Clarkson, A.R., Woodroffe, A.J., Bannister, K.M., Lomax-Smith, J.D., and Aarons, I. 1984. The syndrome of IgA nephropathy. Clin. Nephrol. 21:7-14.

27. Friedberg, M., Larsen, S., and Denneberg, T. 1978 Yersinia enterocolitica and glomerulonephritis. Lancet. 1:498-499.

28. Hubert, D., Beaufils, M., and Meyrier, A. 1984. [Immunoglobulin A glomerular nephropathy associated with inflammatory colitis. Apropos of 2 cases]. Presse Med. 13:1083-1085.

29. Macpherson, A.J., et al. 2000. A primitive T cell-independent mechanism of intestinal mucosal IgA responses to commensal bacteria. Science. 288:2222-2226.

30. Rodicio, J.L. 1984. Idiopathic IgA nephropathy. Kidney Int. 25:717-729.

31. Kamata, T., et al. 2000. Increased frequency of surface IgA-positive plasma cells in the intestinal lamina propria and decreased IgA excretion in hyper IgA (HIGA) mice, a murine model of IgA nephropathy with hyperserum IgA. J. Immunol. 165:1387-1394.

32. Vaerman, J.P., Andre, C., Bazin, H., and Heremans, J.F. 1973. Mesenteric lymph as a major source of serum IgA in guinea pigs and rats. Eur. J. Immunol. 3:580-584.

33. Mackay, F., Majeau, G.R., Lawton, P., Hochman, P.S., and Browning, J.L. 1997. Lymphotoxin but not tumor necrosis factor functions to maintain splenic architecture and humoral responsiveness in adult mice. Eur. J. Immunol. 27:2033-2042.

34. Wagner, N., et al. 1996. Critical role for $\beta 7$ integrins in formation of the gut-associated lymphoid tissue. Nature. 382:366-370.

35. Shaikh, R.B., et al. 2001. Constitutive expression of LIGHT on T cells leads to lymphocyte activation, inflammation, and tissue destruction. J. Immunol. 167:6330-6337.
36. Gomez-Guerrero, C., Gonzalez, E., and Egido, J. 1993. Evidence for a specific IgA receptor in rat and human mesangial cells. J. Immunol. 151:7172-7181.

37. Hendrickson, B.A., et al. 1995. Altered hepatic transport of immunoglobulin A in mice lacking the $\mathrm{J}$ chain. J. Exp. Med. 182:1905-1911.

38. Harper, S.J., Allen, A.C., Pringle, J.H., and Feehally, J. 1996. Increased dimeric IgA producing B cells in the bone marrow in IgA nephropathy determined by in situ hybridisation for J chain mRNA. J. Clin. Pathol. 49:38-42.

39. Harper, S.J., et al. 1995. Increased dimeric IgA-producing B cells in tonsils in IgA nephropathy determined by in situ hybridization for J chain mRNA. Clin. Exp. Immunol. 101:442-448.

40. Layward, L., Finnemore, A.M., Allen, A.C., Harper, S.J., and Feehally, J. 1993. Systemic and mucosal IgA responses to systemic antigen challenge in IgA nephropathy. Clin. Immunol. Immunopathol. 69:306-313.

41. Mackay, F., et al. 1998. Both the lymphotoxin and tumor necrosis factor pathways are involved in experimental murine models of colitis. Gastroenterology. 115:1464-1475.

42. Murai, M., et al. 2003. Peyer's patch is the essential site in initiating murine acute and lethal graft-versus-host reaction. Nat. Immunol. 4:154-160.

43. Ohmacht, C., et al. 1997. Recurrent immunoglobulin A nephropathy after renal transplantation: a significant contributor to graft loss. Transplantation. 64:1493-1496

44. Stevceva, L., Pavli, P., Husband, A.J., and Doe, W.F. 2001. The inflammatory infiltrate in the acute stage of the dextran sulphate sodium induced colitis: B cell response differs depending on the percentage of DSS used to induce it. BMC Clin. Pathol. 1:3.

45. Gommerman, J.L., and Browning, J.L. 2003. Lymphotoxin/light, lymphoid microenvironments and autoimmune disease. Nat. Rev. Immunol. 3:642-655. 